\title{
Implementation outcomes of evidence-based quality improvement for depression in VA community based outpatient clinics
}

\author{
John Fortney ${ }^{1,2,3^{*}}$, Mark Enderle ${ }^{4,9}$, Skye McDougall ${ }^{5}$, Jeff Clothier ${ }^{3,10}$, Jay Otero ${ }^{6}$, Lisa Altman ${ }^{7,8}$ and \\ Geoff Curran ${ }^{1,2,3}$
}

\begin{abstract}
Background: Collaborative-care management is an evidence-based practice for improving depression outcomes in primary care. The Department of Veterans Affairs (VA) has mandated the implementation of collaborative-care management in its satellite clinics, known as Community Based Outpatient Clinics (CBOCs). However, the organizational characteristics of $\mathrm{CBOCs}$ present added challenges to implementation. The objective of this study was to evaluate the effectiveness of evidence-based quality improvement (EBQI) as a strategy to facilitate the adoption of collaborative-care management in CBOCs.

Methods: This nonrandomized, small-scale, multisite evaluation of EBQI was conducted at three VA Medical Centers and 11 of their affiliated CBOCs. The Plan phase of the EBQI process involved the localized tailoring of the collaborative-care management program to each $\mathrm{CBOC}$. Researchers ensured that the adaptations were evidence based. Clinical and administrative staff were responsible for adapting the collaborative-care management program for local needs, priorities, preferences and resources. Plan-Do-Study-Act cycles were used to refine the program over time. The evaluation was based on the RE-AIM (Reach, Effectiveness, Adoption, Implementation, Maintenance) Framework and used data from multiple sources: administrative records, web-based decision-support systems, surveys, and key-informant interviews.
\end{abstract}

Results: Adoption: 69.0\% (58/84) of primary care providers referred patients to the program. Reach: 9.0\% (298/3,296) of primary care patients diagnosed with depression who were not already receiving specialty care were enrolled in the program. Fidelity: During baseline care manager encounters, education/activation was provided to 100\% (298/ 298) of patients, barriers were assessed and addressed for 100\% (298/298) of patients, and depression severity was monitored for $100 \%$ (298/298) of patients. Less than half (42.5\%, 681/1603) of follow-up encounters during the acute stage were completed within the timeframe specified. During the acute phase of treatment for all trials, the Patient Health Questionnaire (PHQ9) symptom-monitoring tool was used at 100\% (681/681) of completed followup encounters, and self-management goals were discussed during 15.3\% (104/681) of completed follow-up encounters. During the acute phase of treatment for pharmacotherapy and combination trials, medication adherence was assessed at 99.1\% (575/580) of completed follow-up encounters, and side effects were assessed at 92.4\% (536/580) of completed follow-up encounters. During the acute phase of treatment for psychotherapy and combination trials, counseling session adherence was assessed at 83.3\% (239/287) of completed follow-up encounters. Effectiveness: 18.8\% (56/298) of enrolled patients remitted (symptom free) and another 22.1\% (66/298) responded to treatment (50\% reduction in symptom severity). Maintenance: $91.9 \%$ (10/11) of the CBOCs chose to sustain the program after research funds were withdrawn.

\footnotetext{
* Correspondence: John.Fortney@va.gov

${ }^{1}$ Health Services Research and Development, Central Arkansas Veterans

Healthcare System, North Little Rock, AR, USA

Full list of author information is available at the end of the article
} 
Conclusions: Provider adoption was good, although reach into the target population was relatively low. Fidelity and maintenance were excellent, and clinical outcomes were comparable to those in randomized controlled trials. Despite the organizational barriers, these findings suggest that EBQI is an effective facilitation strategy for CBOCs.

Trial registration: Clinical trial \# NCT00317018.

\section{Introduction}

Collaborative-care management $(\mathrm{CCM})$ is an evidencebased practice that involves a multidisciplinary depression care team (e.g., primary care providers, nurse care managers, pharmacists, psychologists, psychiatrists) providing guideline-concordant depression treatment in the primary care setting. Numerous effectiveness studies have demonstrated that CCM improves outcomes for primary care patients treated for depression [1-10]. The CCM model has been rolled out nationally in the United States by the Department of Veterans Affairs (VA) Medical Centers as part of the Primary Care/Mental Health Integration Initiative [11].

More recently, the VA has encouraged the implementation of CCM in its Community Based Outpatient Clinics (CBOCs), where $64 \%$ of veterans receive their care [12], and mandated implementation in those categorized as large (5,000-10,000 patients) and very large (> 10,000 patients). Veterans treated at CBOCs have similar demographic characteristics as veterans treated at VA Medical Centers (VAMCs) [13]. All CBOCs provide primary care services, and most large and very large CBOCs also provide specialty mental health services. However, veterans treated in CBOCs have significantly fewer mental health visits than do veterans treated at VAMCs [14]. Twenty-six percent of CBOCs are private clinical organizations contracting with the VA to provide primary care services to veterans [12]. Veterans treated in contract $\mathrm{CBOCs}$ have significantly fewer mental health visits than do veterans treated in VA-staffed (i.e., owned and operated) CBOCs [15]. While CCM could potentially address this disparity, there are numerous barriers to implementing a complex clinical program like CCM in small contract CBOCs.

The organizational characteristics of contract $\mathrm{CBOCs}$ present added challenges to the implementation of CCM. For example, contract CBOCs receive capitated payments (a fixed amount per enrollee to cover a defined scope of services) from the VA and, thus, must consider the financial risk associated with depression quality-improvement efforts. As a result, contract CBOCs may be less willing to comply with VA qualityimprovement initiatives compared to VA-staffed clinics, unless these initiatives are embedded into their legal contracts. In addition, the majority of contract $\mathrm{CBOCs}$ do not have on-site psychiatrists, and because half of contract $\mathrm{CBOCs}$ are located in rural areas, [16] recruiting psychiatrists to small contract $\mathrm{CBOCs}$ is typically not feasible. A previous randomized trial documented that the CCM model can be adapted using telemedicine technologies to effectively improve outcomes for patients treated in CBOCs without on-site psychiatrists [17]. While there is good evidence that telemedicine-based CCM improves outcomes in contract CBOCs, no implementation strategy is known to be effective for this type of organizational context.

The overall goal of our research was to facilitate the adoption of telemedicine-based CCM in contract CBOCs. The Promoting Action on Research in Health Services (PARiHS) framework proposes that successful adoption of an evidence-based practice depends on (1) evidence, (2) context, and (3) facilitation [18]. Evidence includes results from randomized trials, as well as anecdotal evidence from clinical experience $[19,20]$. Context includes both factors internal to the organization, such as culture, climate, and capacity, [21-29] as well as external forces, such as mandates and performance measures. Facilitation typically involves an integrated set of implementation strategies to promote adoption. In this study, we used a facilitation method known as evidencebased quality improvement (EBQI). EBQI has been used successfully to implement CCM in VA Medical Centers [30]. Our specific objective was to test the feasibility of EBQI as an implementation strategy for telemedicinebased CCM in contract CBOCs. Results should inform efforts to roll out complex evidence-based practices to small satellite clinics of integrated healthcare systems.

EBQI was developed by Rubenstein and colleagues based on the findings of the Mental Health Awareness Project, which compared two quality-improvement strategies for depression in primary care [31,32]. Clinics were randomized to either a top-down centralized quality-improvement model or a bottom-up locally driven quality-improvement model [32] The top-down approach involved centralized experts implementing depression evidence-based practices, with some input from local primary care staff. The bottom-up approach involved local clinical staff implementing depression evidence-based practices, with some input from experts. The bottom-up quality-improvement teams had both the best and worst outcomes in terms of fidelity to the evidence base [32]. This finding suggests that the bottom-up approach has the best potential for quality improvement but is subject to substantial variation 
depending on local climate, culture, and capacity [32]. These findings are consistent with two well-designed implementation studies that found that traditional continuous quality-improvement models do not improve depression outcomes [33,34]. Qualitative analyses of the Mental Health Awareness Project also indicated that the top-down approach was more efficient, but the project failed to attain buy-in from local clinicians and administrators [35]. In contrast, the bottom-up quality-improvement approach promoted customization and buy-in but was perceived to be overly time-consuming and inefficient (e.g., reinventing the wheel) [35]. Based on these findings, the EBQI model was developed, which involves both centralized strategic decision making and local tactical decision making [35]. There is a growing consensus among implementation experts [36-38] and frontline clinicians and managers $[32,35,39]$ that quality-improvement strategies that incorporate both top-down and bottom-up approaches hold the most promise for sustained implementation of evidence-based practices.

In EBQI, both researchers (clinical experts, implementation experts) and local staff participate fully in the qualityimprovement process, with the researchers facilitating rather than dictating implementation efforts $[32,35,39]$. Thus, EBQI is intended to foster a researcher/clinician partnership that promotes buy-in from leadership [40,41]. Lack of support from leadership has been shown to be one of the most important barriers to the implementation of the CCM [42]. While emphasizing the involvement of outside experts and empirical evidence, EBQI stresses that an organization's own healthcare professionals and staff are best positioned to improve their systems [40]. Clinicians and administrators contribute the local knowledge needed to tailor the evidence-based practice for their own particular needs and organizational capabilities. Researchers contribute knowledge of the evidence base; ensure fidelity to the evidence base; and supply materials, procedures, and tools needed for successful implementation. In addition to providing expertise, researchers in the EBQI model also facilitate problem solving and provide ongoing technical support for developing data collection/analysis tools, informatics, and training materials. EBQI also emphasizes continuously revising the adapted evidence-based practice based on feedback during Plan-Do-Study-Act cycles and, thus, should lead to adapted evidence-based practices that are robust, user friendly, and feasible to deploy in realworld practice settings. The primary objective of this research was to test the feasibility of EBQI as a facilitation strategy for implementing telemedicine-based CCM in contract CBOCs.

\section{Methods}

This nonrandomized, small-scale, multisite evaluation of EBQI was conducted at three VAMCs and their affiliated CBOCs located in two regional Veterans Integrated Service Networks (VISNs) in the United States. The VAMCs participating in the implementation were chosen based on their number of affiliated contract CBOCs, their willingness to participate, and their potential for success as perceived by VISN leadership. The three VAMCs were affiliated with 11 contract CBOCs.

\section{Implementation strategy}

Each of the three VAMCs had an EBQI team comprised of stakeholders from mental health, primary care, and the CBOCs, as well as the principal investigator. There were no EBQI meetings involving team members from multiple sites, although the principal investigator shared solutions and tools across sites to increase implementation efficiency. Two of the VAMCs chose to have the telemedicine-based CCM program operated by (i.e., administratively housed in) Mental Health, and the third chose to have the program operated by Primary Care. Especially important for this EBQI process was the involvement and buy-in from leadership. Conceptual models of implementation and theories of organizational change strongly emphasize the importance of buy-in from leadership $[20,36,43]$. Therefore, we designed our EBQI process to engage and leverage clinic leadership. However, personnel from the contract CBOCs could not participate in the EBQI process directly because contract CBOCs were not under the purview of an Institutional Review Board and, therefore, could not be directly engaged in research. Instead, the $\mathrm{CBOC}$ liaison at the VAMC represented the view of $C B O C$ stakeholders.

The initial Plan phase of the EBQI process involved the localized tailoring of the telemedicine-based CCM model to each VAMC and their CBOCs. Planning was based on the Steps and Decisions Guide for Implementing Depression CCM Models, which was developed specifically for the study [44]. The CCM experts utilized their experience implementing depression research protocols and their knowledge of the depression and quality-improvement literature to ensure the adaptations to the telemedicine-based CCM model were evidence based. The chiefs of mental health/primary care, depression nurse care managers, other clinical leaders at the VAMC, and the CBOC liaisons were responsible for championing the implementation of the program and adapting the telemedicine-based CCM for local needs, priorities, preferences, and resources (within the fidelity parameters defined by the CCM experts). For example, each $E B Q I$ team developed unique criteria and methods for identifying which patients were ineligible due to substance dependence. In addition, particular attention was devoted to aligning the telemedicine-based CCM program with the VA depression performance measures. Performance measures are reported by the VA Central 
Office on a quarterly basis and determine the salary bonuses of VAMC Directors.

The Do phase at each of the VAMCs involved the initial launch of the program, following a site visit from the depression care manager at the $\mathrm{CBOC}$ considered to have the highest chances for success. Beginning with just one site allowed us to identify and resolve any unforeseen problems before launching the program at other CBOCs. It also allowed us to share any short-term success experienced by this $\mathrm{CBOC}$ with the clinicians at other CBOCs in order to promote provider adoption. Subsequent Do phases involved the launches of the program at the other CBOCs. The Study phase included monthly EBQI conference calls with each site that discussed the implementation efforts. These calls were informed by the data from the Net Decision Support System (NetDSS) depression care manager workload reports that provided information about patient enrollment and fidelity to the care manager protocol (see description below), as well as anecdotal experiences of the care managers and their psychiatrist supervisors. Any implementation problems were discussed during the monthly conference calls and/or via email with the stakeholders. The Act phases of the EBQI process involved the refinement of the adapted telemedicinebased CCM program based on the implementation experiences and data collection described above as the Plan-Do-Study-Act cycle was repeated.

\section{Training and decision-support tools}

The implementation experts also developed training and decision-support tools to promote fidelity to the evidence base. Depression care managers were trained using the VA Mental Health QUERI Depression Care Manager Training Manual, which was specifically developed for the study. A PowerPoint (Microsoft Corporation, Redmond, WA, USA) slide set was also developed to facilitate face-to-face training of the depression care managers. In addition, a web-based decision-support system (NetDSS) and a NetDSS User's Guide were specifically developed for the study to promote care manager fidelity [45]. Difficulty with information technology is one of the most commonly reported barriers to CCM adoption, [42] especially information technology supporting symptom monitoring [46]. NetDSS is based on a highly structured intervention protocol used for a previous effectiveness study of telemedicine-based CCM [19]. NetDSS provides context-specific decision support in real time during patient encounters by guiding care managers through an evidence-based encounter using self-scoring instruments, scripts, and clinical algorithms to identify new medication trials or counseling trials, treatment phases, and outcome milestones, such as treatment response (i.e., $50 \%$ reduction in symptom severity), remission (i.e., symptom free), and relapse. NetDSS is self-documenting and automatically generates a progress note at the end of the encounter, which was copied and pasted into the electronic health record. NetDSS functionality is divided into five categories: (1) panel management, (2) trial management, (3) clinical decision support, (4) progress note generator, and (5) workload/outcomes report generator. NetDSS was continuously revised based on feedback from the depression nurse care managers as part of the Plan-Do-Study-Act process. The NetDSS workload reports were used to provide fidelity and outcomes data to the EBQI teams for the Plan-Do-Study-Act process.

\section{Evaluation}

The evaluation was based on the RE-AIM (Reach, Effectiveness, Adoption, Implementation, Maintenance) Framework [47-50]. Reach represents the absolute number/ proportion of eligible/targeted patients who receive the evidence-based practice [47]. Adoption represents the absolute number/proportion of staff who use the evidence-based practice [47]. Implementation represents the fidelity of the evidence-based practice as implemented in routine care [47]. Effectiveness represents the clinical impact (on patient outcomes) of the evidence-based practice as implemented in routine care settings [49]. Maintenance represents the degree to which the implementation of the evidence-based practice is sustained [47]. To have an impact on health at the population level, an intervention must be adopted by providers, reach a large proportion of the targeted patient population, be implemented with fidelity, effectively improve outcomes, and be maintained after research funds are withdrawn. In addition, we measured implementation costs.

\section{Adoption}

To measure adoption, data were extracted from the Medical SAS Datasets at the Austin Information Technology Center. The post-period was defined as the 12 months after each CBOC start date (defined as the date the first patient was enrolled in the telemedicine-based CCM program), which ranged from April 2006 to February 2008. The number of primary care providers at each $\mathrm{CBOC}$ was determined from the SAS Medical Datasets using unique provider IDs for all types of primary care providers (e.g., general internist, advance practice nurse). The number of primary care providers referring a patient to a depression care manager during the 12-month post-period was identified from NetDSS. The adoption rate for each CBOC was defined as the total number of primary care providers referring a patient to the depression care manager (identified from NetDSS) during the 12-month period divided by the 
total number of primary care providers seeing patients during the 12-month period (identified from SAS Medical Datasets).

\section{Reach}

To measure reach, data were extracted from the SAS Medical Datasets at the Austin Information Technology Center for the 12-month post-period. Index visits for patients during the 12-month post-period were defined as the first primary care encounter at the CBOC with a depression diagnosis. Patients were excluded if they had a specialty mental health visit or a diagnosis of bipolar disorder or schizophrenia during the six months prior to the CBOC start date or if the index visit was a specialty mental health encounter. The number of patients referred to the depression care manager during the 12month post-period was identified from NetDSS. Reach during the 12-month post-period at each CBOC was defined as the total number of patients referred to the depression care manager (identified from NetDSS) divided by the total number of patients with a depression diagnosis who were not already receiving specialty care (identified in the SAS Medical Datasets).

\section{Implementation/fidelity}

To measure fidelity, data were extracted from NetDSS for the 12-month post-period. NetDSS automatically collects data about whether care-manager modules were completed during care-manager encounters. Fidelity during the acute and continuation phases of treatment was reported in aggregate using the NetDSS outcomes reports routine. For the first encounter during the acute phase of treatment, the fidelity measures included the proportion of patients receiving education/activation, barrier assessment/resolution, and Patient Health Questionnaire (PHQ9) depression symptom monitoring. For follow-up encounters during the acute phase of treatment, fidelity measures included the proportion of encounters where self-management goals were discussed, depression symptoms were monitored, medication adherence and sideeffects were assessed (for pharmacotherapy and combination pharmacotherapy/psychotherapy trials only), and counseling adherence was assessed (for psychotherapy and combination trials only). In addition, NetDSS reports what portion of follow-up encounters were completed within the prespecified timeframe: every two weeks in the acute phase for pharmacotherapy and combination pharmacotherapy/psychotherapy trials, every four weeks for watchful waiting and psychotherapy-only trials, and every four weeks for all trials in the continuation phase.

\section{Effectiveness}

To measure effectiveness, data were extracted from the NetDSS final disposition codes for patients enrolled during the 12-month post-period. Final disposition codes were as follows: 1 = remitted and completed the continuation, 2 responded ( $>50 \%$ reduction in PHQ9 score) and completed the continuation phase without relapsing, 3 = unable to complete baseline assessment or lost to follow-up, $4=$ disenrolled at patient's request, 5 $=$ disenrolled at provider's request, 6 = became ineligible (e.g., entered a nursing home or moved away), and $7=$ referred to a higher level of care (e.g., specialty mental health) due to lack of response or detection of complex psychiatric comorbidities.

\section{Maintenance}

Maintenance represents the sustainability of the telemedicine-based CCM program and the extent to which it has been institutionalized into the organization's practices and policies. Maintenance was measured using two complementary instruments approximately a year after the last $\mathrm{CBOC}$ enrolled its first patient into the telemedicine-based CCM program, when research funds were no longer supporting the salary of clinical personnel. The Level of Use interview [51] measured sustained use of the program, and the Level of Institutionalization survey [52] measured the degree to which the program was institutionalized within the organization. Level of Use was measured using key informant interviews with the Medical Directors of each of the 11 CBOCs and the Chief of Mental Health or Chief of Primary Care at the VAMC (depending on which service line operated the program). Using a structured interview guide and inductive questioning, the Level of Use framework classified the CBOCs into eight ranked levels of adoption according to their adoption behaviors. The first three levels distinguish between stages of nonuse (nonuse, orientation, and preparation). The next five levels distinguish between stages of use (mechanical, routine, refinement, integration, renewal), and these distinctions are made based on the type of adaptations or refinements that are being made to the innovation. Level of Institutionalization was measured via telephone survey of the Chief of Mental Health or Chief of Primary Care at the VAMC (depending on which service line operated the program). Institutionalization implies that the organization has modified itself to incorporate the innovation and that the innovation has ceased to become novel and has been embedded in standard operating procedures. The Level of Institutionalization instrument measures an innovation's institutionalization among four subsystems: production, maintenance, supportive, and managerial. The production subsystem is responsible for delivering clinical services; to be institutionalized, the innovation must be integrated with other routine clinical services. The maintenance subsystem represents personnel; to be institutionalized, the innovation must be supported by 
permanent employees. The supportive subsystem represents external organizational forces; to be institutionalized, the innovation must have a stable source of funding and permanent office space. The managerial subsystem represents the executive and supervisory functions; to be institutionalized, the innovation must be assigned to a specific service, staff must have written job descriptions, and performance measures and progress reports must be required. For each subsystem, the Level of Institutionalization survey asks the respondent about the degree to which the organization has institutionalized the innovation (e.g., supported by permanent employees), and the responses are averaged to calculate an overall mean for each subsystem. The Level of Institutionalization instrument has three levels for each subsystem: low institutionalization (mean score $\leq 2$ ), moderate institutionalization $(2<$ mean score $\leq 3)$ and high institutionalization (mean score $>3$ ).

\section{Pre-implementation planning and fixed implementation costs}

Pre-implementation planning costs represent the cost that a VAMC would incur to implement a telemedicinebased CCM model in CBOCs. The total cost of attending EBQI meetings was calculated by multiplying the number of meetings each EBQI member attended by their hourly wage estimated from their grade and step (or nurse level). The total cost of implementation activities between EBQI meetings was calculated by multiplying the estimated hours per month devoted to implementation activities by the hourly wage estimated from grade and step (or nurse level). Fixed implementation costs represent the one-time cost of implementing telemedicine-based CCM, including the development of the VA Mental Health QUERI Depression Care Manager Training Manual, NetDSS (web-based decision support system), and the NetDSS User's Guide. These costs were calculated by estimating the number of hours worked on each of these activities and from final budget expenditures. These fixed costs would not be incurred for VAMCs implementing the telemedicine-based CCM model in CBOCs in the future.

The research was approved by the Institutional Review Boards of the Central Arkansas Veterans Healthcare System, the Greater Los Angeles Healthcare System, and the Loma Linda Healthcare System.

\section{Results}

\section{Characteristics of contract community based outpatient} clinics

The characteristics of the 11 participating CBOCs are presented in Table 1. During the 12-month post-period, 42,330 unique patients were treated at the 11 CBOCs. The number of patients ranged from 1,325 to 7,411 across the CBOCs, placing the clinics in the small (< 1,500 patients), medium (1,500-5,000 patients), and large (5,000-10,000 patients) categories. On average, the percent of patients diagnosed with depression (but without a specialty mental health visit or a diagnosis of bipolar disorder or schizophrenia) ranged from $4.5 \%$ to $12.3 \%$, with an average of $7.8 \%$ across the CBOCs. Among this group of patients eligible for depression care management $(n=3,296)$, the number of depression-related primary care encounters per patient during the 12-month post-period ranged from 1.1 to 1.8 , with an average of 1.3 (standard deviation $[\mathrm{SD}]=0.6$ ), which was about two-thirds of the total number of primary care encounters. Also among this group, the number of depression-related specialty mental health visits during the 12-month post-period ranged from 0.3 to 1.4 , with an average of $0.5(\mathrm{SD}=1.8)$, which accounted for about half of the total number of specialty mental health visits.

\section{Evidence-based quality-improvement process}

An average of 11.7 (range = 9-14) staff members participated in at least one EBQI meeting at each of the affiliated VAMCs, but only 4.7 (range = 3-6) participated in five or more EBQI meetings on average. The duration of the EBQI process averaged 21.3 months (range $=16-25$ ) across the three VAMCs, and the average number of EBQI meetings was 13.7 (range = 13-15). Of those participating in five or more meetings, the average number of hours per month spent on implementation efforts was 13.3 (range $=5.5-19.6$ ). Of the EBQI team members, the depression nurse care managers spent the most hours per month on implementation efforts $($ mean $=28.7$, range $=16-45)$.

\section{Collaborative-care management program structure}

All three VAMCs chose to include one depression nurse care manager available by telephone and one supervising tele-psychiatrist on the CCM team. The two programs that were operated by Mental Health were able to obtain psychiatric supervision for the depression care managers more easily than the site operated by Primary Care. Other types of providers (e.g., pharmacists, psychotherapists) were not included on any of the CCM teams. Each VAMC chose to pilot the telemedicinebased CCM program at one CBOC first and then spread the program to other CBOCs affiliated with the VAMC. All three VAMCs chose to enroll only patients who were referred to the care manager by their primary care provider. In contrast, none of the VAMCs chose to target all patients screening positive for depression, or all patients diagnosed with depression, or all patients initiating antidepressant treatment. However, all three VAMCs did choose to use an existing Depression Case Finder to identify patients targeted by VA depression 
Table 1 Patterns of depression treatment during the 12-month post-implementation period by study site

\begin{tabular}{|c|c|c|c|c|c|c|}
\hline Facility & $\begin{array}{l}\text { Unique } \\
\text { number of } \\
\text { patients }\end{array}$ & $\begin{array}{l}\text { Unique number of } \\
\text { patients diagnosed } \\
\text { with depression }\end{array}$ & $\begin{array}{c}\text { Mean primary } \\
\text { care visits per } \\
\text { patient }\end{array}$ & $\begin{array}{l}\text { Mean primary care } \\
\text { visits for depression } \\
\text { per patient }\end{array}$ & $\begin{array}{l}\text { Mean specialty } \\
\text { mental health visits } \\
\text { per patient }\end{array}$ & $\begin{array}{l}\text { Mean specialty mental } \\
\text { health visits for } \\
\text { depression per patient }\end{array}$ \\
\hline \multicolumn{7}{|l|}{$\begin{array}{l}\text { VAMC } \\
\text { A }\end{array}$} \\
\hline $\begin{array}{l}\mathrm{CBOC} \\
1\end{array}$ & 3474 & $\begin{array}{c}243 \\
(7.0 \%)\end{array}$ & 2.0 & 1.2 & 0.8 & 0.4 \\
\hline $\begin{array}{l}\mathrm{CBOC} \\
2\end{array}$ & 1968 & $\begin{array}{c}112 \\
(5.7 \%)\end{array}$ & 1.9 & 1.3 & 1.2 & 0.8 \\
\hline $\begin{array}{l}\mathrm{CBOC} \\
3\end{array}$ & 4534 & $\begin{array}{c}487 \\
(10.7 \%)\end{array}$ & 1.7 & 1.3 & 0.8 & 0.3 \\
\hline $\begin{array}{l}\mathrm{CBOC} \\
4\end{array}$ & 1325 & $\begin{array}{c}163 \\
(12.3 \%)\end{array}$ & 2.1 & 1.8 & 1.8 & 1.4 \\
\hline \multicolumn{7}{|l|}{$\begin{array}{l}\text { VAMC } \\
\text { B }\end{array}$} \\
\hline $\begin{array}{l}\mathrm{CBOC} \\
5\end{array}$ & 4545 & $\begin{array}{c}203 \\
(4.5 \%)\end{array}$ & 1.9 & 1.2 & 1.6 & 0.4 \\
\hline $\begin{array}{l}\mathrm{CBOC} \\
6\end{array}$ & 5341 & $\begin{array}{c}431 \\
(8.1 \%)\end{array}$ & 1.7 & 1.2 & 1.1 & 0.6 \\
\hline $\begin{array}{l}\mathrm{CBOC} \\
7\end{array}$ & 7411 & $\begin{array}{c}673 \\
(9.1 \%)\end{array}$ & 1.6 & 1.3 & 0.8 & 0.4 \\
\hline $\begin{array}{l}\mathrm{CBOC} \\
8\end{array}$ & 2374 & $\begin{array}{c}162 \\
(6.8 \%)\end{array}$ & 1.8 & 1.3 & 2.0 & 0.9 \\
\hline $\begin{array}{l}\mathrm{CBOC} \\
9\end{array}$ & 3642 & $\begin{array}{c}182 \\
(5.0 \%)\end{array}$ & 1.8 & 1.1 & 0.9 & 0.5 \\
\hline \multicolumn{7}{|l|}{$\begin{array}{l}\text { VAMC } \\
\text { C }\end{array}$} \\
\hline $\begin{array}{l}\text { CBOC } \\
10\end{array}$ & 3252 & $\begin{array}{c}279 \\
(8.6 \%)\end{array}$ & 2.0 & 1.3 & 1.1 & 0.6 \\
\hline $\begin{array}{l}\text { CBOC } \\
11\end{array}$ & 4464 & $\begin{array}{c}361 \\
(8.1 \%)\end{array}$ & 2.4 & 1.4 & 1.0 & 0.6 \\
\hline Total & 42,330 & $\begin{array}{l}3,296 \\
(7.8 \%)\end{array}$ & $\begin{array}{c}1.9 \\
(\mathrm{SD}=1.2)\end{array}$ & $\begin{array}{c}1.3 \\
(S D=0.6)\end{array}$ & $\begin{array}{c}1.1 \\
(S D=4.7)\end{array}$ & $\begin{array}{c}0.5 \\
(\mathrm{SD}=1.8)\end{array}$ \\
\hline
\end{tabular}

VAMC = Department of Veterans Affairs Medical Center; $C B O C=$ Community Based Outpatient Clinic; $S D=$ standard deviation.

$V A=$ Department of Veterans Affairs; $C B O C=$ Community Based Outpatient Clinic.

performance measures (e.g., those with a new diagnosis and a new antidepressant prescription) and to request consults (i.e., provider referrals) to the program for these patients. In addition, care manager clinic codes, diagnoses, and Current Procedural Terminology (CPT) codes were chosen to ensure that care manager encounters contributed to the VA depression follow-up visit performance measure. All three VAMCs chose to exclude patients with serious mental illnesses, as well as patients already receiving (or eventually referred) to specialty mental health. However, in actuality, care managers sometimes continued to provide care management to patients referred to their supervising tele-psychiatrist for ongoing care. Care management activities at all three VAMCs included education/activation, barrier assessment/resolution, symptom monitoring, medication adherence monitoring, side-effects monitoring, and selfmanagement. All of the VAMCs chose to exclude brief counseling as a care manager activity to maximize the caseload capacity of the care manager. None of the VAMCs developed formal guidelines (e.g., decision nodes) for referring patients from the telemedicinebased CCM program to specialty mental health (i.e., to a higher level of care), but rather, this decision was left to the discretion of the care manager's supervising telepsychiatrist.

\section{Adoption}

There were 84 primary care providers who diagnosed one or more patients with depression at the implementation CBOCs during the 12-month post-period. Approximately 69.0\% (58/84) of these providers referred at least one patient to the depression care manager. Adoption rates ranged across CBOCs from a low of $33.3 \%$ to a high of $100 \%$ (see Figure 1 ).

\section{Reach}

There were 3,296 patients diagnosed with depression (but without a prior specialty mental health visit or a diagnosis of bipolar disorder or schizophrenia) at implementation CBOCs during the 12-month post-period. Nine percent $(298 / 3296)$ of these patients had an 


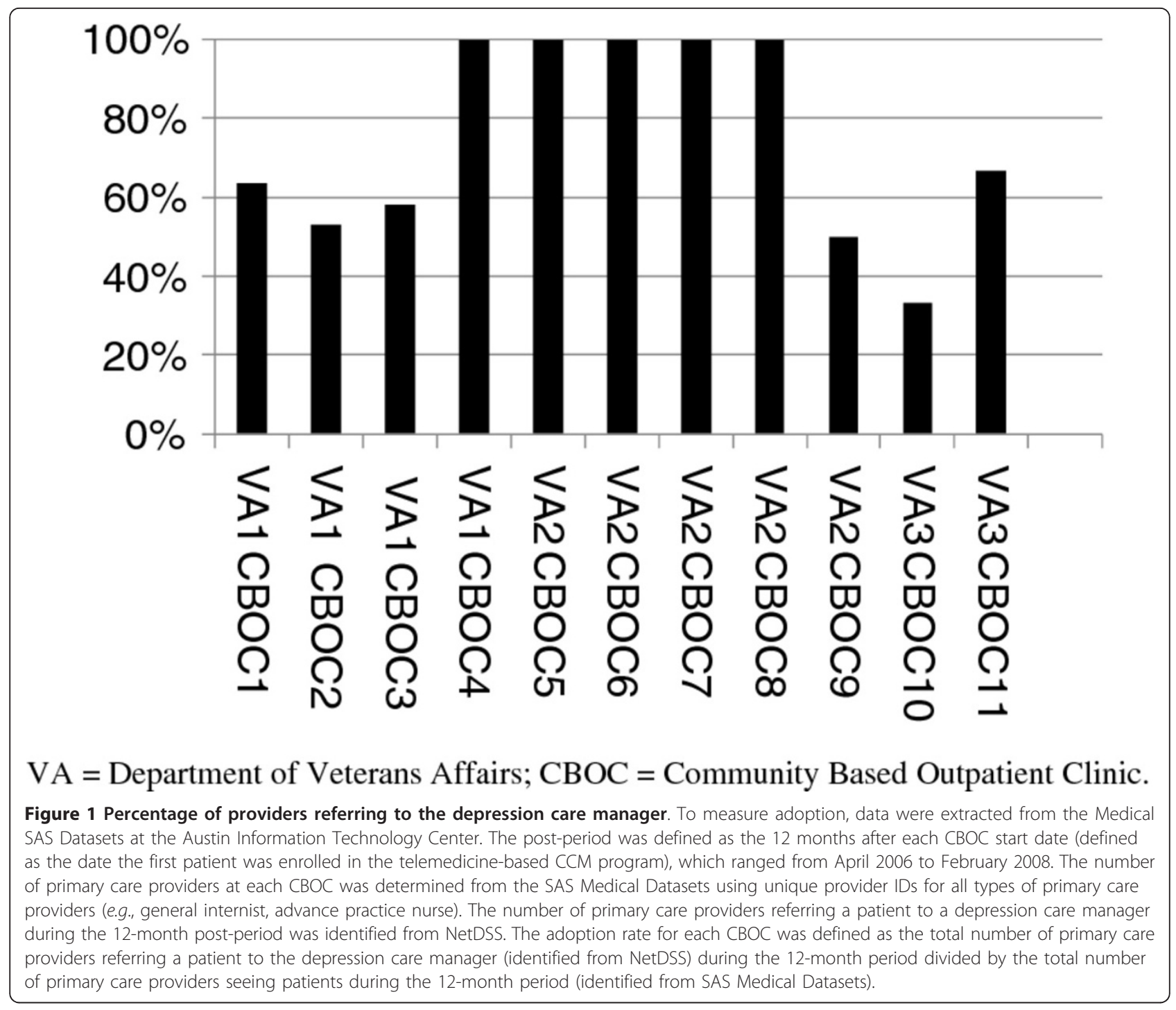

encounter with a depression care manager. Reach ranged across CBOCs from a low of $1.1 \%$ to a high of 49.1\% (see Figure 2).

\section{Implementation/fidelity}

For most domains, fidelity to the care manager protocol was excellent for the 298 patients who had encounters with a depression care manager. Patient education/activation and barriers assessment/resolution were provided at $100 \%(298 / 298)$ of baseline encounters (i.e., the first care manager encounter). Likewise, the PHQ9 depression symptom-monitoring tool was used to assess depression severity at $100 \%(298 / 298)$ of baseline encounters. The 298 patients had 368 treatment trials in the acute phase. Almost half $(48.4 \%, 178 / 368)$ of the acute phase trials were pharmacotherapy only, $28.8 \%(106 / 368)$ were combination pharmacotherapy/psychotherapy, $6.3 \%$ (23/368) were psychotherapy only, and $16.6 \%(61 / 368)$ were watchful waiting. Less than half $(42.5 \%, 681 / 1603)$ of follow-up encounters during the acute stage were completed within the timeframe specified in the acute phase of treatment (i.e., within two or four weeks, depending on the trial type). During the acute phase of treatment for all trials, the PHQ9 symptom-monitoring tool was used at $100 \%(681 / 681)$ of completed follow-up encounters, and self-management goals were discussed during $15.3 \%(104 / 681)$ of completed follow-up encounters. During the acute phase of treatment for pharmacotherapy and combination trials, medication adherence was assessed at $99.1 \%(575 / 580)$ of completed follow-up encounters, and side effects were assessed at 92.4\% (536/ 580) of completed follow-up encounters. During the acute phase of treatment for psychotherapy and combination trials, counseling-session adherence was assessed 


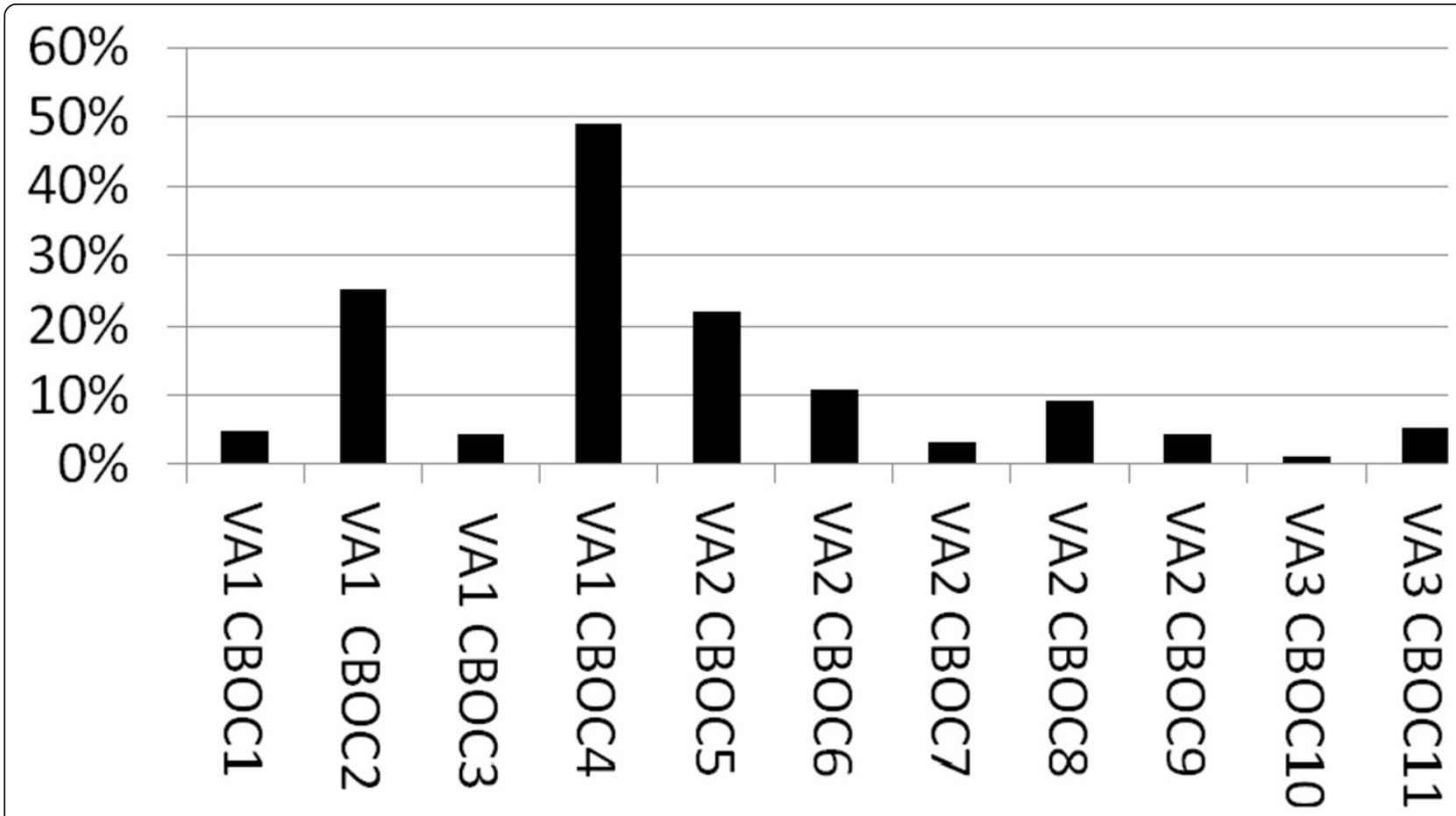

\section{$\mathrm{VA}=$ Department of Veterans Affairs; CBOC $=$ Community Based Outpatient Clinic.}

Figure 2 Percentage of patients referred to the depression care manager. To measure reach, data were extracted from the SAS Medical Datasets at the Austin Information Technology Center for the 12-month post-period. Index visits for patients during the 12-month post-period were defined as the first primary care encounter at the $\mathrm{CBOC}$ with a depression diagnosis. Patients were excluded if they had a specialty mental health visit or a diagnosis of bipolar disorder or schizophrenia during the six months prior to the $\mathrm{CBOC}$ start date or if the index visit was a specialty mental health encounter. The number of patients referred to the depression care manager during the 12-month post-period was identified from NetDSS. Reach during the 12-month post-period at each $\mathrm{CBOC}$ was defined as the total number of patients referred to the depression care manager (identified from NetDSS) divided by the total number of patients with a depression diagnosis who were not already receiving specialty care (identified in the SAS Medical Datasets).

at $83.3 \%(239 / 287)$ of completed follow-up encounters. During the continuation phase of treatment, $54.6 \%$ (189/ 346) of follow-up encounters were completed within the prespecified timeframe (i.e., within four weeks).

\section{Effectiveness}

Depression care managers enrolled 298 patients into the telemedicine-based CCM program. Of these, 7.4\% (22/ 298 ) could not be reached for a baseline evaluation and another $8.7 \%$ (26/298) were lost to follow-up. In addition, 9.7\% (29/298) were disenrolled at the patient's request and $0.3 \%(1 / 298)$ were disenrolled at the primary care provider's request. Another $7.7 \%$ (23/298) became ineligible (e. g., moved out of area), and $24.2 \%$ (72/298) were referred to a higher level of care (e.g., referred to specialty mental health). Overall, 18.8\% (56/298) remitted (symptom free) and completed the continuation phase of treatment and another $22.1 \%(66 / 298)$ responded to treatment and completed the continuation phase without relapsing. Thus, $40.9 \%(122 / 298)$ of enrolled patients had positive outcomes because they either remitted or responded and completed without relapsing. Examining outcomes for completers only $(n=194), 34.0 \%(66 / 194)$ responded to treatment and completed, $28.8 \%(56 / 194)$ remitted and completed (62.8\% positive outcomes, $122 / 194)$, and $37.1 \%$ $(72 / 194)$ were referred to a higher level of care.

\section{Maintenance}

There was variation across the 11 CBOCs on the Level of Use instrument. One (9.1\%) CBOC was classified as a nonuser (level 1), two (18.2\%) were classified as mechanical users (level 3), one (9.1\%) was classified as a refinement user (level 6), and seven (63.6\%) were classified as integrated users (level 7) (see Figure 3). Level of Institutionalization was mostly in the high to moderate range across all three VAMCs (see Figure 4).

\section{Pre-implementation planning costs and fixed costs}

The total cost of attending EBQI meetings was $\$ 8,270.90$. The total costs for the time spent on implementation 


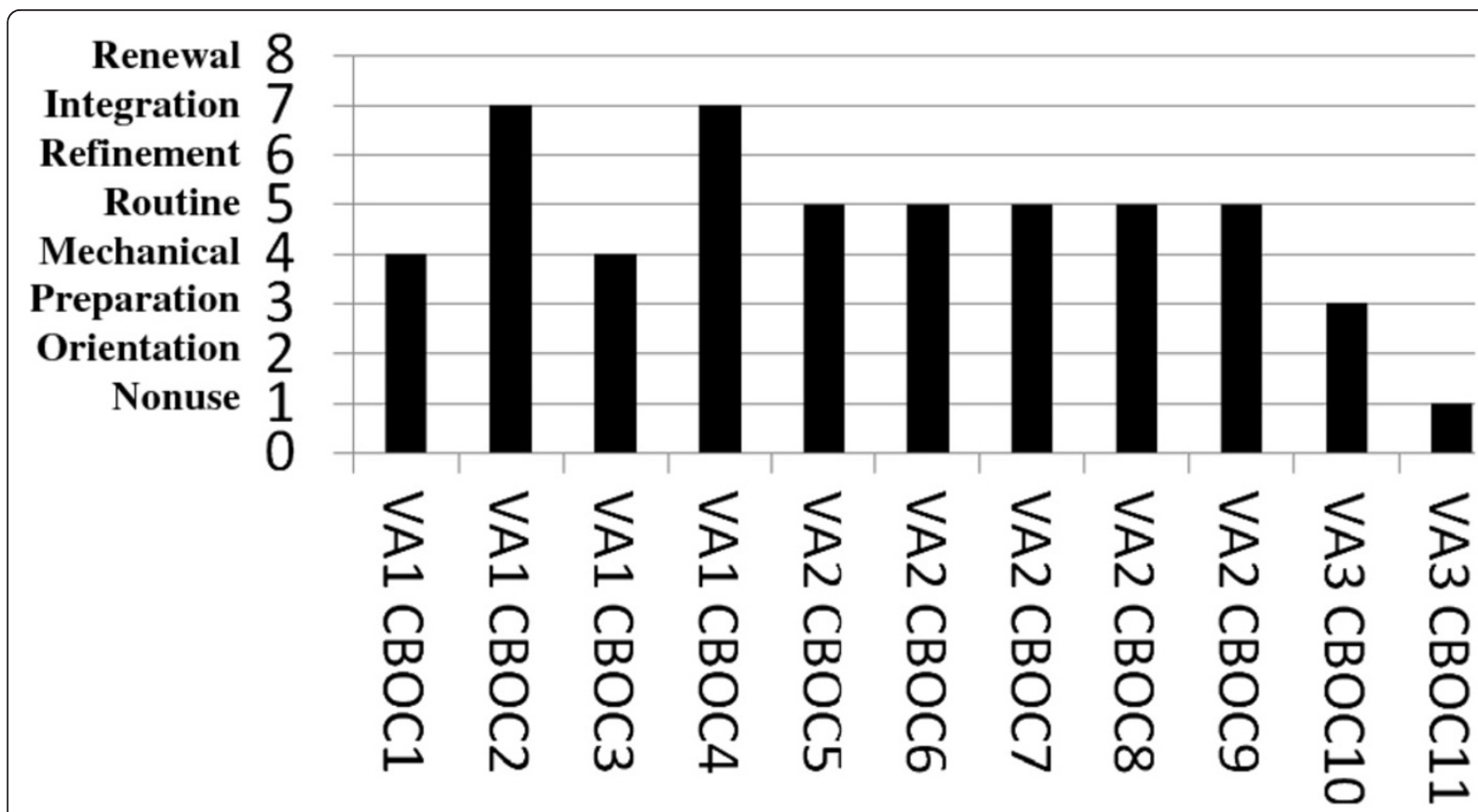

\section{$\mathrm{VA}=$ Department of Veterans Affairs $; \mathrm{CBOC}=$ Community Based Outpatient Clinic.}

Figure 3 Level of use of telemedicine-based collaborative-care management. The Level of Use interview measured sustained use of the program and was administered approximately a year after the last CBOC enrolled its first patient into the telemedicine-based CCM program, when research funds were no longer supporting the salary of clinical personnel. Level of Use was measured using key informant interviews with the Medical Directors of each of the 11 CBOCs and the Chief of Mental Health or Chief of Primary Care at the VAMC (depending on which service line operated the program). Using a structured interview guide and inductive questioning, the Level of Use framework classified the CBOCs into eight ranked levels of adoption according to their adoption behaviors. The first three levels distinguish between stages of nonuse (nonuse, orientation, and preparation). The next five levels distinguish between stages of use (mechanical, routine, refinement, integration, renewal), and these distinctions are made based on the type of adaptations or refinements that are being made to the innovation.

between EBQI meetings was $\$ 84,483.45$. The total preimplementation planning costs across all three VAMCs were estimated to be $\$ 92,753.79$. Dividing total pre-implementation planning costs by the 3,296 patients diagnosed with depression at implementation CBOCs and targeted by the telemedicine-based CCM program during the 12 month post-period yields a total pre-implementation planning cost per targeted patient of $\$ 28.14$. Dividing total pre-implementation planning costs by the 298 patients enrolled in the telemedicine-based CCM program during the 12-month post-period yields a total pre-implementation planning cost per patient reached of $\$ 311.25$. The fixed cost for developing the Mental Health QUERI Depression Care Manager Training Manual was \$17,182. The fixed cost of developing NetDSS was $\$ 100,370$. The fixed cost for developing the NetDSS User's Guide was $\$ 12,391$. The total fixed costs were $\$ 129,943$.

\section{Discussion}

The telemedicine-based CCM program had an excellent adoption rate by primary care providers (69\%), although reach into the target patient population was relatively low overall (9\%). The low proportion of targeted patients reached by the program is somewhat difficult to interpret, as it is not clear what proportion of patients with depression would have benefited clinically from depression care management. Moreover, because the EBQI teams specifically chose to target those patients whose primary care providers thought would benefit from the telemedicine-based CCM program, it made it impossible to identify in a systematic manner what the true denominator was for the reach evaluation. Presumably, many of the patients diagnosed with depression (used as the dominator for the reach evaluation) were in remission or stable on medications during the time period and would not have benefited clinically from the program. Of those enrolled in the telemedicine-based CCM program, fidelity to the care manager protocol was excellent. The high level of fidelity can be attributed, in large part, to the use of the NetDSS care manager decision support system. NetDSS workload/ outcomes reports were discussed at EBQI team meetings 


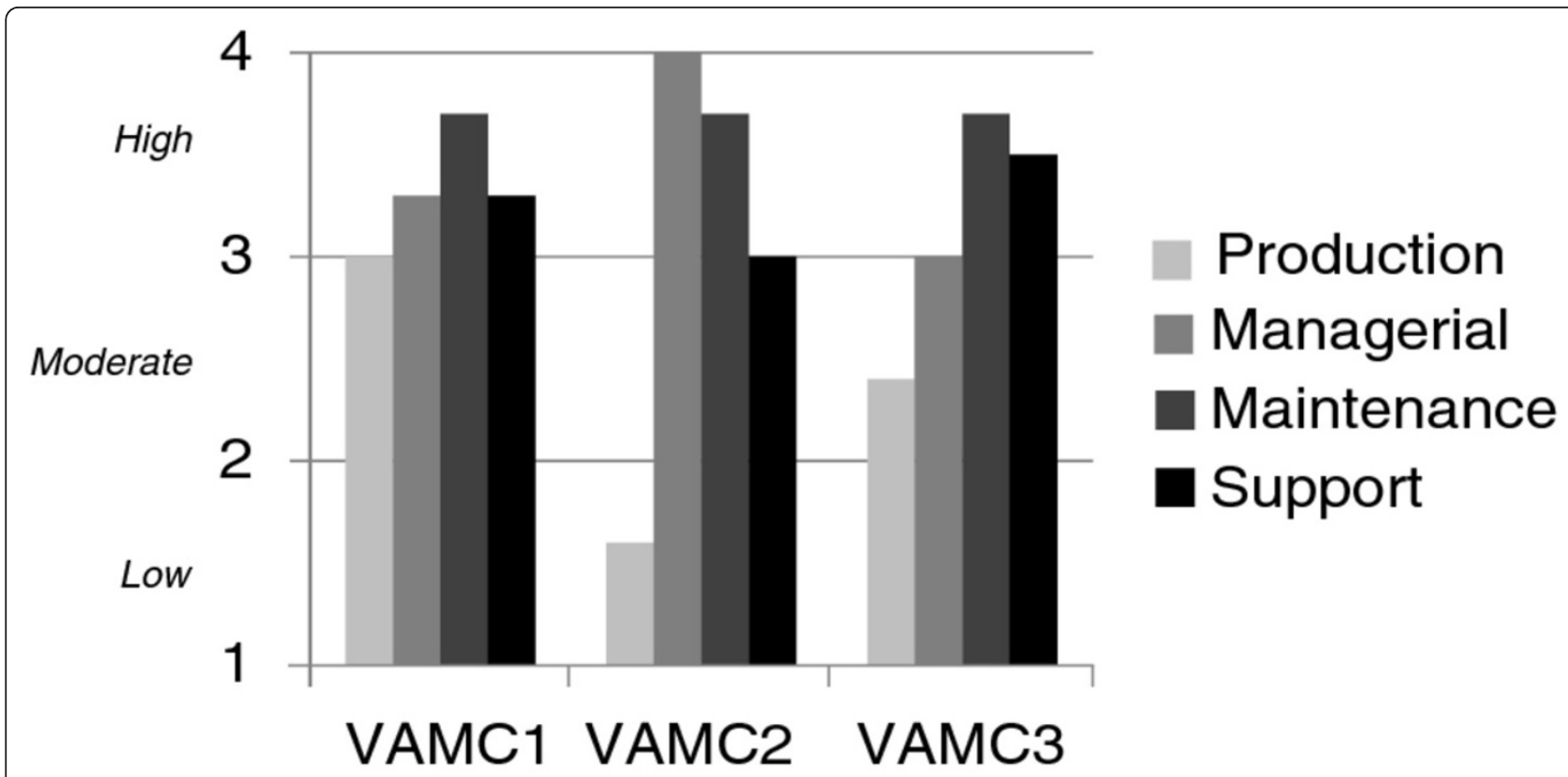

VAMC $=$ Department of Veterans Affairs Medical Center.

Figure 4 Level of institutionalization of telemedicine-based collaborative care management. Level of Institutionalization survey measured the degree to which the program was institutionalized within the organization. Level of Institutionalization was measured via telephone survey of the Chief of Mental Health or Chief of Primary Care at the VAMC (depending on which service line operated the program). Institutionalization implies that the organization has modified itself to incorporate the innovation and that the innovation has ceased to become novel and has been embedded in standard operating procedures. The Level of Institutionalization instrument measures an innovation's institutionalization among four subsystems: production, maintenance, supportive, and managerial. The production subsystem is responsible for delivering clinical services; to be institutionalized, the innovation must be integrated with other routine clinical services. The maintenance subsystem represents personnel; to be institutionalized, the innovation must be supported by permanent employees. The supportive subsystem represents external organizational forces; to be institutionalized, the innovation must have a stable source of funding and permanent office space. The managerial subsystem represents the executive and supervisory functions; to be institutionalized, the innovation must be assigned to a specific service, staff must have written job descriptions, and performance measures and progress reports must be required. For each subsystem, the Level of Institutionalization survey asks the respondent about the degree to which the organization has institutionalized the innovation (e.g., supported by permanent employees), and the responses are averaged to calculate an overall mean for each subsystem. The Level of Institutionalization instrument has three levels for each subsystem: low institutionalization (mean score $\leq 2)$, moderate institutionalization $(2<$ mean score $\leq 3)$ and high institutionalization (mean score $>3$ ).

and used to address problems with care manager fidelity. The biggest problems with fidelity were completing assessments within the prespecified timeframe and care manager activities that were not required to be completed by NetDSS (i.e., the optional self-management planning). In addition, the clinical outcomes of enrolled patients were comparable to intervention patients in a prior randomized effectiveness study of telemedicinebased CCM [19]. Of those enrolled, 18.8\% completed the continuation phase in remission and another $22.1 \%$ responded to treatment and completed the continuation phase without relapsing. Thus, from an intent-to-treat perspective, $40.9 \%$ of patients enrolled in telemedicinebased CCM had positive outcomes, which is somewhat higher than the 12-month intent-to-treat response rate (36\%) reported in a previous randomized effectiveness trial of telemedicine-based CCM that enrolled patients screening positive for depression [19]. A previous study has demonstrated that patients referred to CCM by their primary care provider as part of routine care experience greater symptom improvement than those randomized to CCM after being enrolled in a research study using traditional recruitment methods [53]. For those completing the telemedicine-based CCM program, $65.8 \%$ either remitted or responded without relapsing. Importantly, the telemedicine-based CCM programs continued to be used in a sustained manner after research funds were withdrawn. Likewise, the telemedicine-based CCM programs became institutionalized into the operations of the affiliated VAMCs.

Based on these findings, we conclude that EBQI is a feasible facilitation strategy for contract CBOCs. This finding is significant because there were many barriers to adoption in contract $\mathrm{CBOCs}$, and these results are a 
testament to the strength of this particular facilitation method. Rogers has argued that innovation complexity is a barrier to diffusion, $[54,55]$ and telemedicine-based CCM is a multifaceted intervention that involves a multidisciplinary care team and, thus, is relatively complex to put into practice. Rogers also argued that incompatibility can be a barrier to adoption, $[54,55]$ and telemedicinebased CCM was not compatible with traditional referralbased treatment patterns of depression treatment in the VA. Because the VA is an integrated system of care, referrals from primary care to specialty mental health care are common, even for mild to moderate depression. Because CCM encourages a more integrated approach to care, it was somewhat disruptive to put into practice. However, there was anecdotal evidence that implementing telemedicine-based CCM in CBOCs was less disruptive than implementing practice-based $C C M$ in parent VAMCs. This may have been due to the long travel distance to specialty mental healthcare for CBOC patients, which may have resulted in the $\mathrm{CBOC}$ providers being less reliant on referrals than primary care providers at parent VAMCs. The EBQI process also overcame other barriers to diffusion identified by Rogers, especially trialability $[54,55]$. Trialability is the degree to which an innovation can be tested on a limited basis and was a barrier to the adoption because patients can remain in care management for 6 to 12 months. Piloting telemedicine-based CCM in the Do phase facilitated the ability to "test drive" the intervention.

As discussed in the PARiHS framework, context is also an important factor to the adoption of evidencebased practices [20]. With respect to external contextual factors, the sustained implementation of telemedicinebased CCM was facilitated by the VA's national Primary Care/Mental Health Integration Initiative, which concurrently promoted the implementation of CCM models. Even though CCM implementation was only mandated for the one large $\mathrm{CBOC}$ in our sample, the implementation of the telemedicine-based CCM program was highly compatible $[54,55]$ with the existing priorities of the VA Central Office. In contrast to the favorable external context of implementing telemedicine-based CCM, the internal context of contract $\mathrm{CBOCs}$ was a barrier to implementation. Contract CBOCs are likely to have very different organizational cultures and climates than VAMCs and VA-staffed CBOCs. Likewise, because contract $\mathrm{CBOCs}$ receive capitated payments, they have a financial incentive to minimize costs, including both patient care expenses and the investment of slack resources in quality-improvement efforts. Although empirical evidence indicates that telemedicine-based CCM does not increase primary care visits or costs [54], the additional cost of collaborating clinically with an off-site care team may have been a concern of the primary care providers. This concern may have led to fewer referrals, thereby contributing to the low level of reach into the target population.

It is also important to note that the EBQI implementation strategy was relatively resource intensive. Ignoring the fixed cost of developing the training materials and decision support system, the pre-implementation planning cost per patient reached was $\$ 311$. It required the commitment of a large number of busy administrators and clinicians, as well as the involvement of implementation researchers. Therefore, the EBQI implementation strategy used in this study is probably not particularly scalable. However, assuming that the sites included in this study are generalizable to other CBOCs, it is probably not necessary to repeat our relatively resourceintensive adaption process to spread the telemedicinebased CCM to other sites [55]. Presumably, much of the spread to other CBOCs could be more standardized than it was in this study. A more scalable (i.e., less intensive) EBQI strategy could also be employed to support spread, perhaps involving larger groups of $\mathrm{CBOCs}$ in the Plan-Do-Study-Act cycles.

An important limitation of this study is that VAMCs were chosen based on their willingness to participate and their potential for success as perceived by VISN leadership. While the results from these implementation sites may not be generalizable to all VAMCs, it is advisable to initially choose sites that are committed to implementation for small-scale, multisite evaluation studies. Similar to an efficacy study, the purpose of smallscale, multisite evaluation is to determine whether implementation strategies can work under favorable circumstances [56]. Generalizability of sites becomes a more critical issue for region-wide demonstration studies, where the purpose is to determine whether promising implementation strategies are effective under a wide range of contexts [56]. A second limitation was that we did not have the resources to collect qualitative data about provider adoption and patient reach. Qualitative data obtained from key informant interviews with frontline providers would have helped us better interpret our quantitative findings about adoption and reach. Another limitation of the study was that, due to lack of Institutional Review Board oversight at contract CBOCs, onsite providers and administrators could not be engaged in research and, therefore, could not participate directly in the EBQI process. This was an artificial barrier to implementation that we encountered in the context of conducting research that would not be an issue outside this context. Finally, this feasibility study had no comparison group that used an alternative implementation strategy, and thus, we only demonstrated that EBQI can be successful in this context, not that it was more effective than other implementation strategies. 
The major strength of the evaluation component of the study was the integration of clinical information obtained from administrative data and the decision support system (NetDSS) to measure adoption, reach, implementation/fidelity, and effectiveness. Likewise, another strength was measuring maintenance by integrating quantitative and qualitative data obtained from the Level of Institutionalization survey and the Level of Use structured key-informant interviews. The major strength of the implementation component of the study was development and refinement of the decision support system (NetDSS), which substantially enhanced care manager fidelity. The use of NetDSS has spread to nonstudy sites at one of the regional VISNs and is now being used for other mental health disorders. Based on the results of a previous effectiveness study and the implementation tools developed for this study, telemedicine-based CCM is now listed on the National Registry of Evidence-based Programs and Practices (NREPP), a service of the Substance Abuse and Mental Health Services Administration (SAMHSA). NREPP is a searchable database of interventions, and SAMHSA has developed this resource to help agencies and organizations implement evidence-based mental health practices in their communities.

\section{Conclusions}

The results of this study suggest that EBQI can be used successfully to implement a complex disruptive intervention (telemedicine-based CCM) with fidelity, despite the organizational barriers in contract CBOCs. The success of the EBQI process was likely due to the partnership between clinical leaders and researchers. The researchers were able to develop evidence-based training materials and decision-support tools that seemed to promote fidelity and effectiveness. In turn, the clinical leaders were able to align the telemedicine-based CCM program with the needs of CBOC staff and patients, which seemed to promote adoption, reach, and maintenance. Given that EBQI is resource intensive, it may be a particularly appropriate facilitation strategy during the early stages of an implementation initiative when a relatively small number of sites are committed to successful implementation.

\footnotetext{
Acknowledgements

We would like to thank the depression care managers who participated in this study: William Raney, Susan Lyrla, and Sue Donovan. We would also like to thank Cheryl Hardcastle, Kathy Henderson, Amanda Lunsford, Michael McCarther, Debbie Mittman, Lisa Rubenstein, Barbara Simon, Susan Vivell, and James Williams for their important contributions to the study. This research was supported by VA Quality Enhancement Research Initiative (QUERI) IMV 04-360 grant to Drs. Fortney (PI), Enderle (Co-PI), and McDougall (Co-PI); the VA HSR\&D Center for Mental Health and Outcomes Research; the VA South Central Mental IIIness Research Education and Clinical Center; and the VA HSR\&D Center for the Study of Healthcare Provider Behavior.
}

\section{Author details}

${ }^{1}$ Health Services Research and Development, Central Arkansas Veterans Healthcare System, North Little Rock, AR, USA. ${ }^{2}$ South Central Mental IIIness Research Education and Clinical Center, Central Arkansas Veterans Healthcare System, North Little Rock, AR, USA. ${ }^{3}$ Department of Psychiatry, University of Arkansas for Medical Sciences, Little Rock, AR, USA. ${ }^{4}$ Office of Quality and Safety, Department of Veterans Affairs, Washington, DC, USA. ${ }^{5}$ Desert Pacific Healthcare Network (VISN 22), Long Beach, CA, USA. ${ }^{6}$ Behavioral Medicine Service, VA Loma Linda Healthcare System, Loma Linda, CA, USA. ${ }^{7}$ Primary/ Ambulatory Care, Greater Los Angeles Healthcare System, Los Angeles, CA, USA. ${ }^{8}$ Department of Medicine, University of California, Los Angeles, CA, USA ${ }^{9}$ At the time the study was conducted, Dr. Enderle was affiliated with the South Central Veterans Healthcare Network (VISN 16. ${ }^{10}$ At the time the study was conducted, Dr. Clothier was affiliated with the Mental Health Service, Central Arkansas Healthcare System.

\section{Authors' contributions}

JF obtained funding and contributed to study conceptualization and study design, acquisition of data, statistical analysis, interpretation of data, and drafting of the manuscript. ME and SM obtained funding, contributed to study conceptualization and study design, and critically revised the manuscript for important intellectual content. JC, JO, and LA supervised the clinical team, provided administrative leadership, and critically revised the manuscript for important intellectual content. GC helped with study conceptualization and study design, collection of Level of Use data from key-informant interviews, interpretation of data, drafting of manuscript, and critical revision of the manuscript for important intellectual content. All authors read and approved the final manuscript.

\section{Competing interests}

The authors declare that they have no competing interests.

Received: 30 July 2011 Accepted: 11 April 2012 Published: 11 April 2012

\section{References}

1. Katon W, Von Korff M, Lin E, Simon G, Walker E, Unützer J, et al: Stepped collaborative care for primary care patients with persistent symptoms of depression: a randomized trial. Arch Gen Psychiatry 1999, 56(12):1109-1115.

2. Katon W, Robinson P, Von Korff M, Lin E, Bush T, Ludman E, et al: A multifaceted intervention to improve treatment of depression in primary care. Arch Gen Psychiatry 1996, 53(10):924-932.

3. Simon GE, VonKorff M, Rutter C, Wagner E: Randomised trial of monitoring, feedback, and management of care by telephone to improve treatment of depression in primary care. Br Med J 2000, 320(7234):550-554.

4. Rost K, Nutting P, Smith J, Werner J, Duan N: Improving depression outcomes in community primary care practice: a randomized trial of the QuEST intervention. J Gen Intern Med 2001, 16(3):143-149.

5. Wells KB, Sherbourne C, Schoenbaum M, Duan N, Meredith L, Unützer J, et al: Impact of disseminating quality improvement programs for depression in managed primary care: a randomized controlled trial. $J$ Am Med Assoc 2000, 283(2):212-220.

6. Finley PR, Rens HR, Pont JM, Gess SL, Louie C, Bull SA, et al: Impact of collaborative care model upon depression in primary care: a randomized controlled trial. Pharmacotherapy 2003, 23:1175-1185.

7. Adler DA, Bungay KM, Wilson IB, Pei Y, Supran S, Peckham E, et al: The impact of a pharmacist intervention on 6-month outcomes in depressed primary care patients. Gen Hosp Psychiatry 2004, 26(3):199-209.

8. Unützer J, Katon W, Callahan CM, Williams JW Jr, Hunkeler E, Harpole L, et al: Collaborative care management of late-life depression in the primary care setting: a randomized controlled trial. J Am Med Assoc 2002, 288(22):2836-2845.

9. Hedrick SC, Chaney EF, Felker B, Liu CF, Hasenberg N, Heagerty P, et al: Effectiveness of collaborative care depression treatment in Veteran's Affairs primary care. J Gen Intern Med 2003, 18(1):9-16.

10. Badamgarav E, Weingarten SR, Henning JM, Knight K, Hasselblad V, Gano A $\mathrm{Jr}$, et al: Effectiveness of disease management programs in depression: a systematic review. Am J Psychiatry 2003, 160(12):2080-2090.

11. Post EP, Metzger M, Dumas $P$, Lehmann L: Integrating mental health into primary care within the Veterans Health Administration. Fam Syst Health 2010, 28(2):83-90. 
12. Panangala SV, Mendez BHP: Veterans Health Administration: CommunityBased Outpatient Clinics. Congressional Research Service 2010, R41044, Ref Type: Report.

13. Fortney JC, Borowsky SJ, Hedeen AN, Maciejewski ML, Chapko MK: VA community-based outpatient clinics: access and utilization performance measures. Med Care 2002, 40(7):561-569.

14. Maciejewski ML, Perkins M, Li YF, Chapko M, Fortney JC, Liu CF: Utilization and expenditures of Veterans obtaining primary care in community clinics and VA medical centers: an observational cohort study. BMC Health Serv Res 2007, 7:56.

15. Liu CF, Chapko MK, Perkins MW, Fortney J, Maciejewski ML: The impact of contract primary care on health care expenditures and quality of care. Med Care Res Rev 2008, 65(3):300-314.

16. Chapko M, Borowsky S, Fortney J, Hedeen A, Hoegle M, Maciejewski M, et al: Evaluation of the Department of Veterans Affairs Community-Based Outpatient Clinics. Med Care 2002, 40(7):555-560.

17. Fortney J, Pyne JM, Edlund MJ, Williams DK, Robinson DE, Mittal D, et al: A randomized trial of telemedicine-based collaborative care for depression. J Gen Intern Med 2007, 22(8):1086-1093.

18. Kitson A, Harvey G, McCormack B: Enabling the implementation of evidence based practice: a conceptual framework. Qual Health Care 1998, 7(3):149-158.

19. Greenhalgh T: Intuition and evidence-uneasy bedfellows? $\mathrm{Br} J$ Gen Pract 2002, 52:395-400.

20. Haynes RB: What kind of evidence is it that Evidence-Based Medicine advocates want health care providers and consumers to pay attention to? BMC Health Serv Res 2002, 2:3.

21. Parker LE, Fickel JJ, Yano EM, Simon C, Bonner LM, Ritchie MJ, et al: Organizational context and adoption of new clinical practices 2009, Ref Type: Unpublished Work.

22. Steckler A, Goodman RM, Kegler MC: Mobilizing organizations for health enhancement: Theories of organizational change. In Health Behavior and Health Education: Theory, Research and Practice. Edited by: Glanz K, Rimmer BK, Lewis FM. San Francisco, CA: Jossey-Bass; 2002:335-360.

23. Glisson C, Green P: The effects of organizational culture and climate on the access to mental health care in child welfare and juvenile justice systems. Administration and Policy in Mental Health and Mental Health Services Research 2006, 33(4):433-448.

24. Owen RR, Hudson T, Thrush C, Thapa P, Armitage T, Landes RD: The effectiveness of guideline implementation strategies on improving antipsychotic medication management for schizophrenia. Med Care 2008, 46(7):686-691.

25. Glisson C: The organizational context of children's mental health services. Clin Child Fam Psychol Rev 2002, 5(4):233-253.

26. Glisson C, James LR: The cross-level effects of culture and climate in human service teams. J Organ Behav 2002, 23:767-794.

27. Schutte K, Yano EM, Kilbourne AM, Wickrama B, Kirchner JE, Humphreys K: Organizational contexts of primary care approaches for managing problem drinking. J Subst Abuse Treat 2009, 36(4):435-445, Epub 2008 Nov 12.

28. Rubenstein LV, Chaney EF, Ober S, Felker B, Sherman SE, Lanto A, et al: Using evidence-based quality improvement methods for translating depression collaborative care research into practice. Family, Systems \& Health 2010, 28(2):91-113.

29. Rost KM, Duan N, Rubenstein LV, Ford DE, Sherbourne CD, Meredith LS, et al: The Quality Improvement for Depression collaboration: general analytic strategies for a coordinated study of quality improvement in depression care. Gen Hosp Psychiatry 2001, 23(5):239-253.

30. Rubenstein LV, Parker LE, Meredith LS, Altschuler A, de Pillis E, Hernandez J, et al: Understanding Team-based Quality Improvement for Depression in Primary Care. Health Serv Res 2002, 37(4):1009-1029.

31. Goldberg HI, Wagner EH, Fihn SD, Martin DP, Horowitz CR, Christensen DB, et al: $\mathrm{A}$ randomized controlled trial of $\mathrm{CQ}$ teams and academic detailing: can they alter compliance with guidelines? Jt Comm J Qual Improv 1998, 24(3):130-142.

32. Solberg LI, Fischer LR, Wei F, Rush WA, Conboy KS, Davis TF, et al: A CQI intervention to change the care of depression: a controlled study. Eff Clin Pract 2001, 4(6):239-249.

33. Parker LE, de Pillis E, Altschuler A, Rubenstein LV, Meredith LS: Balancing participation and expertise: A comparison of locally and centrally managed health care quality improvement within primary care practices. Qual Health Res 2007, 17(9):1268-1279.

34. Greenhalgh T, Robert G, Macfarlane F, Bate P, Kyriakidou O: Diffusion of innovations in service organizations: systematic review and recommendations. Milbank Q 2004, 82(4):581-629.

35. Ginsburg LR, Lewis S, Zackheim L, Casebeer A: Revisiting interaction in knowledge translation. Implementation Science 2007, 2:34.

36. Stange KC, Goodwin MA, Zyzanski SJ, Dietrich AJ: Sustainability of a practice-individualized preventive service delivery intervention. Am J Prev Med 2003, 25:296-300.

37. Parker LE, Kirchner JE, Bonner LM, Fickel JJ, Ritchie MJ, Simons CE, et al: Creating a quality-improvement dialogue: utilizing knowledge from frontline staff, managers, and experts to foster health care quality improvement. Qual Health Res 2009, 19(2):229-242.

38. Rubenstein LV, Mittman BS, Yano EM, Mulrow CD: From understanding health care provider behavior to improving health care: the QUERI framework for quality improvement. Quality Enhancement Research Initiative. Med Care 2000, 38(6 Suppl 1):1129-1141.

39. Mendel P, Meredith LS, Schoenbaum M, Sherbourne CD, Wells KB: Interventions in organizational and community context: a framework for building evidence on dissemination and implementation in health services research. Admin Policy Ment Health 2008, 35(1-2):21-37.

40. Meredith LS, Mendel P, Pearson M, Wu S, Joyce G, Straus JB, et al: Implementation and maintenance of quality improvement for treating depression in primary care. Psychiatr Serv 2006, 57(1):48-55.

41. Rogers EM: Diffusion of Innovations. New York, NY: The Free Press, A Division of Simon \& Schuster, Inc.;, 52003.

42. Fortney JC, Pyne JM, Smith JL, Curran GM, Otero JM, Enderle MA, et al: Steps for implementing collaborative care programs for depression. Popul Health Manag 2009, 12(2):69-79.

43. Fortney JC, Pyne JM, Steven CA, Williams JS, Hedrick RG, Lunsford AK, et al: A web-based clinical decision support system for depression care management. Am J Manag Care 2010, 16(11):849-854.

44. Franx G, Meeuwissen JA, Sinnema H, Spijker J, Huyser J, Wensing M, et al: Quality improvement in depression care in the Netherlands: the depression breakthrough collaborative. A quality improvement report. International Journal of Integrated Care 2009, 9:e84.

45. Glasgow RE, Vogt TM, Boles SM: Evaluating the public health impact of health promotion interventions: the RE-AIM framework. Am J Public Health 1999, 89(9):1322-1327.

46. Glasgow RE, McKay HG, Piette JD, Reynolds KD: The RE-AIM framework for evaluating interventions: what can it tell us about approaches to chronic illness management? Patient Educ Couns 2001, 44(2):119-127.

47. Glasgow RE: Translating research to practice: lessons learned, areas for improvement, and future directions. Diab Care 2003, 26(8):2451-2456.

48. Glasgow RE, Lictenstein E, Marcus AC: Why don't we see more translation of health promotion research to practice? Rethinking the efficacy-toeffectiveness transition. Am J Public Health 2003, 93(8):1261-1267.

49. Hall G, Loucks S, Rutherford W, Newlove B: Levels of use of the innovation: a framework for analyzing innovation adoption. J Teach Educ 1975, 26(1):52-56.

50. Goodman RM, McLeroy KR: Development of level of institutionalization scales for health promotion programs. Health Educ Q 1993, 20(2):161-179.

51. Chaney EF, Rubenstein LV, Liu CF, Yano EM, Bolkan C, Lee M, et al: Implementing collaborative care for depression treatment in primary care: a cluster randomized evaluation of a quality improvement practice redesign. Implement Sci 2011, 6:121.

52. Rogers EM: Diffusion of Innovations. New York: The Free Press, A Divison of Simon \& Schuster, Inc.; 31983.

53. Rogers EM: Diffusion of Innovations. 4 edition. New York, NY: The Free Press; 1995.

54. Fortney JC, Maciejewski M, Tripathi S, Deen TL, Pyne JM: A budget impact analysis of telemedicine-based collaborative care for depression. Med Care

55. Smith JL, Williams JW Jr, Owen RR, Rubenstein LV, Chaney E: Developing a national dissemination plan for collaborative care for depression: QUERI series. Implement Sci 2008, 3:59.

56. Stetler CB, Mittman BS, Francis J: Overview of the VA Quality Enhancement Research Initiative (QUERI) and QUERI theme articles: QUERI Series. Implement Sci 2008, 3(8):1-9. 
doi:10.1186/1748-5908-7-30

Cite this article as: Fortney et al:: Implementation outcomes of evidence-based quality improvement for depression in VA community based outpatient clinics. Implementation Science 2012 7:30.

Submit your next manuscript to BioMed Central and take full advantage of:

- Convenient online submission

- Thorough peer review

- No space constraints or color figure charges

- Immediate publication on acceptance

- Inclusion in PubMed, CAS, Scopus and Google Scholar

- Research which is freely available for redistribution

Submit your manuscript at www.biomedcentral.com/submit 